\title{
X-Ray Photoelectron Spectroscopy
}

National Cancer Institute

\section{Source}

National Cancer Institute. X-Ray Photoelectron Spectroscopy. NCI Thesaurus. Code C78871.

A quantitative spectroscopic technique that both determines the identities and percent composition of elements in a material and measures the elemental chemical and electronic states for a sample. The process, which occurs in a vacuum, involves the irradiation of the material of interest with a beam of aluminum or magnesium X-rays while measuring both the kinetic energy and number of electrons that are released from the outer surface. 